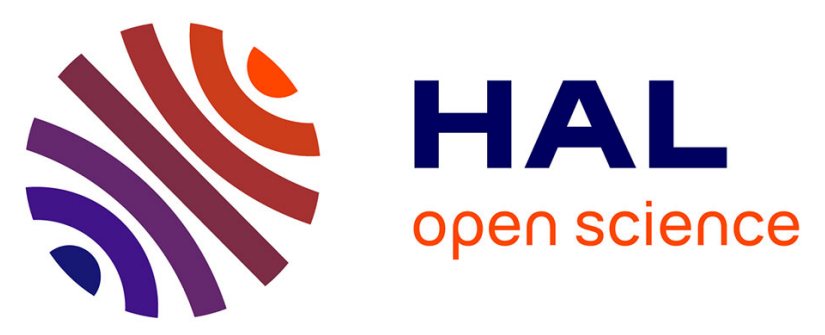

\title{
Molecular Genetic Tools and Emerging Synthetic Biology Strategies to Increase Cellular Oil Content in Chlamydomonas reinhardtii
}

Fantao Kong, Yasuyo Yamaoka, Takeshi Ohama, Youngsook Lee, Yonghua Li-Beisson

\section{To cite this version:}

Fantao Kong, Yasuyo Yamaoka, Takeshi Ohama, Youngsook Lee, Yonghua Li-Beisson. Molecular Genetic Tools and Emerging Synthetic Biology Strategies to Increase Cellular Oil Content in Chlamydomonas reinhardtii. Plant and Cell Physiology, 2019, 60 (6), pp.1184-1196. 10.1093/pcp/pcz022 . hal-02095649

\section{HAL Id: hal-02095649 \\ https://hal.science/hal-02095649}

Submitted on 17 Feb 2020

HAL is a multi-disciplinary open access archive for the deposit and dissemination of scientific research documents, whether they are published or not. The documents may come from teaching and research institutions in France or abroad, or from public or private research centers.
L'archive ouverte pluridisciplinaire HAL, est destinée au dépôt et à la diffusion de documents scientifiques de niveau recherche, publiés ou non, émanant des établissements d'enseignement et de recherche français ou étrangers, des laboratoires publics ou privés. 
archives-ouvertes

\section{Molecular Genetic Tools and Emerging Synthetic Biology Strategies to Increase Cellular Oil Content in Chlamydomonas reinhardtii}

Fantao Kong, Yasuyo Yamaoka, Takeshi Ohama, Youngsook Lee, Yonghua Li-Beisson

\section{To cite this version:}

Fantao Kong, Yasuyo Yamaoka, Takeshi Ohama, Youngsook Lee, Yonghua Li-Beisson. Molecular Genetic Tools and Emerging Synthetic Biology Strategies to Increase Cellular Oil Content in Chlamydomonas reinhardtii. Plant and Cell Physiology, Oxford University Press (OUP), 2019, 60 (6), pp.1184-1196. 10.1093/pcp/pcz022 . hal-02095649

\section{HAL Id: hal-02095649 \\ https://hal.archives-ouvertes.fr/hal-02095649}

Submitted on 17 Feb 2020

HAL is a multi-disciplinary open access archive for the deposit and dissemination of scientific research documents, whether they are published or not. The documents may come from teaching and research institutions in France or abroad, or from public or private research centers.
L'archive ouverte pluridisciplinaire HAL, est destinée au dépôt et à la diffusion de documents scientifiques de niveau recherche, publiés ou non, émanant des établissements d'enseignement et de recherche français ou étrangers, des laboratoires publics ou privés. 


\section{Plant and Cell Physiology}

\section{Molecular Genetic Tools and Emerging Synthetic Biology \\ Strategies to Increase Cellular Oil Content in Chlamydomonas reinhardtii}

\begin{tabular}{|r|l|}
\hline Journal: & Plant and Cell Physiology \\
\hline Manuscript ID & Draft \\
\hline Manuscript Type: & Special Issue - Mini Review \\
\hline Duthor: & n/a \\
\hline Complete List of Authors: & $\begin{array}{l}\text { Kong, Fantao; Pohang University of Science and Technology, Department } \\
\text { of Integrative Bioscience \& Biotechnology; Dalian University of } \\
\text { Technology, School of Life Sciences and Biotechnology } \\
\text { Yamaoka, Yasuyo; Pohang University of Science and Technology, } \\
\text { Department of Integrative Bioscience \& Biotechnology } \\
\text { Ohama, Takeshi; KUT, School of Environmental Science and Engineering } \\
\text { Lee, Youngsook; Pohang University of Science and Technology, life } \\
\text { Science } \\
\text { Li-Beisson, Yonghua ; CEA, Plant Biology and Environmental Microbiology }\end{array}$ \\
\hline Keywords: & $\begin{array}{l}\text { Chlamydomonas reinhardtii, Genome Editing, Metabolic Engineering, Oil } \\
\text { content, Transgene Expression, Triacylglycerol }\end{array}$ \\
\hline
\end{tabular}


Title: Molecular Genetic Tools and Emerging Synthetic Biology Strategies to Increase Cellular Oil Content in Chlamydomonas reinhardtii

Short title: Synthetic Biology to Increase Chlamydomonas Oil

Correspondence to: F. Kong, Department of Integrative Bioscience \& Biotechnology, Pohang University of Science and Technology, Pohang 37673, Korea. Tel: +82 54279 2296; fax: +82 54279 2199; Email: kongfantaohit@,163.com

Subject areas: (4) proteins, enzymes and metabolism; (11) new methodology.

Number of black and white figures (2); colour figure (1); table (1). 


\section{Molecular Genetic Tools and Emerging Synthetic Biology Strategies to Increase Cellular Oil Content in Chlamydomonas reinhardtii}

Fantao Kong ${ }^{1,2^{*}}$, Yasuyo Yamaoka ${ }^{1}$, Takeshi Ohama ${ }^{3}$, Youngsook Lee ${ }^{1,4}$, Yonghua LiBeisson $^{5}$

${ }^{1}$ Department of Integrative Bioscience \& Biotechnology, Pohang University of Science and Technology, Pohang 37673, Korea

${ }^{2}$ School of Life Sciences and Biotechnology, Dalian University of Technology, Dalian 116024, China

${ }^{3}$ School of Environmental Science and Engineering, Kochi University of Technology (KUT), Tosayamada, Kochi 782-8502, Japan

${ }^{4}$ Department of Life Science, Pohang University of Science and Technology, Pohang 37673, Korea

5 Aix-Marseille Univ., CEA, CNRS, BIAM, UMR7265, CEA Cadarache, Saint-Paul-lez Durance F-13108, France

*: Correspondence to Fantao Kong (kongfantaohit@,163.com)

There are no conflicts of interest.

Abbreviation: amiRNA, artificial microRNA; CRISPR, clustered regularly interspaced short palindromic repeats; FA, fatty acid; FP, fluorescent protein; gRNA, guide RNA; N, nitrogen; RNAi, RNA interference; RNPs, ribonucleoproteins; S, sulfur; TAG, triacylglycerol; TALEN, transcription activator-like effector nuclease; TF, transcription factor; ZFN, zinc finger nuclease 


\begin{abstract}
:
Microalgae constitute a highly diverse group of eukaryotic and photosynthetic microorganisms that have developed extremely efficient systems for harvesting and transforming solar energy into energy-rich molecules such as lipids. Although microalgae are considered to be one of the most promising platforms for the sustainable production of liquid oil, the oil content of these organismis is naturally low, and algal oil production is currently not economically viable. Chlamydomonas reinhardtii (Chlamydomonas) is an established algal model due to its fast growth, high transformation efficiency, and well-understood physiology and to the availability of detailed genome information and versatile molecular tools for this organism. In this review, we summarize recent advances in the development of genetic manipulation tools for Chlamydomonas, from gene delivery methods to state-of-theart genome-editing technologies and fluorescent dye-based high-throughput mutant screening approaches. Furthermore, we discuss practical strategies and toolkits that enhance transgene expression, such as choice of expression vector and background strain. We then provide examples of how advanced genetic tools have been used to increase oil content in Chlamydomonas. Collectively, the current literature indicates that microalgal oil content can be increased by overexpressing key enzymes that catalyze lipid biosynthesis, blocking lipid degradation, silencing metabolic pathways that compete with lipid biosynthesis, and modulating redox state. The tools and knowledge generated through metabolic engineering studies should pave the way for developing a synthetic biological approach to enhance lipid productivity in microalgae.
\end{abstract}

Key words: Chlamydomonas reinhardtii, Genome Editing, Metabolic Engineering, Oil content, Transgene Expression, Triacylglycerol 


\section{Introduction}

Microalgae represent a polyphyletic group of photosynthetic eukaryotic microorganisms, and are sources of a number of value-added products, including proteins, pigments, carbohydrates, carotenoids, vitamins, anti-oxidants, and polyunsaturated very long chain fatty acids (Chew et al. 2017). Due to their high biomass productivity and diverse ecological habitats, microalgae have recently been considered an alternative sustainable resource for biofuels. However, the sustainable industrial production of oil, i.e., triacylglycerols (TAGs), from any microalgal species has yet to be realized, due to bottlenecks at both the biological and biotechnological levels. One of the most challenging issues is to increase oil productivity, because most microalgae accumulate large amounts of oil only when subjected to stress conditions (e.g., nutrient depletion), when cell growth is usually impaired (Merchant et al. 2012, Siaut et al. 2011). Therefore, current research is intensively focused on deciphering the molecular mechanisms underlying lipid biosynthesis and the relationship between lipid biosynthesis and biomass growth (Hu et al. 2008, Kong et al. 2018a).

Among microalgal species, the unicellular eukaryotic green alga Chlamydomonas reinhardtii (hereafter, Chlamydomonas) is a well-established algal model, due to its haploid genotype, fast growth, and sequenced genome (Merchant et al. 2007). Chlamydomonas is commonly used to study a number of biological processes, including photosynthesis and flagella-mediated motility. Over the past decade, spurred on by a renewed interest in microalgal oil, Chlamydomonas has emerged as a model organism for studies of lipid metabolism (Du and Benning, 2016, Li-Beisson et al. 2015).

Furthermore, the emergence of synthetic biology provides an opportunity to expedite the development of Chlamydomonas and other microalgal species as a useful industrial biotechnology platform. Synthetic biology, which is based on a combination of standardized biological components (e.g., promoters, terminators, and selection markers), predictive modeling, and iterative design and testing, was first used in bacterial systems and has since advanced to eukaryotic systems, including algae (Crozet et al. 2018). A modular cloning toolkit, comprising 119 functionally validated genetic parts, was developed for Chlamydomonas based on Golden Gate cloning with standard syntax (Crozet et al. 2018). This toolkit has established Chlamydomonas as the next chassis for sustainable synthetic biology.

Here, we present an overview of molecular approaches, such as metabolic engineering and gene disruption strategies, that enable the genetic manipulation of microalgae with the 
aim of identifying metabolic pathways and elucidating physiological mechanisms. We provide examples of successful efforts to genetically manipulate Chlamydomonas to increase oil content, and discuss future challenges and directions in microalgal synthetic biology.

\section{Gene delivery tools and selection systems for Chlamydomonas}

\section{Transformation methods}

Various techniques have been established to deliver DNA into the Chlamydomonas nucleus, including biolistics, electroporation, Agrobacterium-mediated transformation, or even simply vortexing with glass beads, silicon carbide whiskers, or 3-aminopropyl-functionalized magnesium (reviewed by Jinkerson and Jonikas 2015). Biolistic transformation was the first method developed for this purpose and was mediated by microprojectiles, such as tungsten powder and gold particles, and, more recently, by size-controlled mesoporous $\mathrm{TiO}_{2}$ nanoparticles, which are effective at lower density and are less prone to aggregation than are tungsten or gold particles (Hou et al. 2013). Biolistic methods can be used to transform nuclear, chloroplastic, and mitochondrial genomes. This method is efficient but expensive due to the requirement for specialized equipment. Consequently, cheaper and simpler methods (e.g., Agrobacterium-mediated transformation and electroporation) have been developed. Agrobacterium-mediated transformation yields a slightly lower proportion of transgenesilenced transformants than does electroporation, but does not provide significant advantages over electroporation-mediated transformation in Chlamydomonas, due to its labor-intensive nature and large number of false positives (Mini et al. 2018). Thus, electroporation is currently one of the most efficient methods for delivering DNA into the nuclei of cells (Shimogawara et al. 1998, Yamano et al. 2013).

\section{Selectable markers and reporter genes}

Several antibiotic resistance genes have been developed for nuclear or chloroplast transformation in Chlamydomonas. Among these, aadA, encoding an aminoglycoside 3'adenylyltransferase (spectinomycin and streptomycin resistance), is widely used in both nuclear and chloroplast transformation (Meslet-Cladière and Vallon 2011). The GC-rich ble gene, conferring resistance to zeocin, is an effective selectable marker for efficient transgene expression when fused to the target gene (Fuhrmann et al. 1999, Plucinak et al. 2015), but the underlying mechanism is not well understood. It is possible that bleomycin resistance proteins (BLEs) function by sequestering zeocin in a one-to-one ratio, but not through enzymatic 
inactivation (Rasala et al. 2013). However, the use of ble as a selectable maker is limited, because zeocin is light sensitive and may function as a mutagen when applied at high concentrations (Lin et al. 2017). This prompted the development of other selectable markers, such as aphVIII and aph7", which confer paromomycin and hygromycin-resistance, respectively. AphVIII is currently one of the most widely used selectable marker in Chlamydomonas, because it confers a stable drug-resistant phenotype (Meslet-Cladière and Vallon 2011).

In addition to selectable markers, reporter genes have also become important tools in microalgal genetic engineering. For instance, to monitor gene expression and protein localization in vivo, various fluorescent proteins covering most fluorescent ranges (i.e., yellow YFP, blue mTagBFP, cyan mCerulean, green CrGFP, yellow Venus, orange tdTomato, and red mCherry) have been adapted for optimal expression in Chlamydomonas (Neupert et al. 2009, Rasala et al. 2013).

\section{Forward genetics in Chlamydomonas}

\section{Mutagenesis}

Mutagenesis mediated by mutagens (e.g, ethylmethanesulfonate) has been used to generate a library of Chlamydomonas mutants that was subjected to forward genetic screens for altered oil content (Xie et al. 2014, Lee et al. 2014). However, a major drawback of using these mutagens is that point mutations are randomly produced, making it challenging to identify the causative gene(s) for the phenotype.

Random insertional mutagenesis of Chlamydomonas using drug resistance cassettes, which randomly integrate exogenous DNA into the nuclear genome, has been used to generate tens of thousands of transformants in numerous forward genetics studies. Insertional mutants have been generated at large scale and screened for phenotypes related to lipid metabolism, motility, and photosynthesis (Cagnon et al. 2013, Cheng et al. 2017, Dent et al. 2015). An advantage of this approach is that insertional mutations can be mapped without the need for extensive genetic crosses.

\section{Examples of medium-to-high-throughput screening developed for Chlamydomonas}

Oil content can be estimated by staining with fluorescent dyes (e.g., Nile red and BODIPY) and can be combined flow cytometry analysis for high-throughput studies. This strategy has 
been used to screen for Chlamydomonas mutants with altered oil content (Cagnon et al. 2013, Cheng et al. 2017, Tsai et al. 2014). Fluorescence-activated cell sorting of cells that have been stained with Nile red allows for the efficient screening of tens of thousands of Chlamydomonas mutants (Terashima et al. 2015, Xie et al. 2014). This method also allows for quantification of lipid content while preserving cell viability, as Nile red staining does not hinder growth, and requires only five weeks from mutagenesis to mutant isolation (Terashima et al. 2015).

Another approach commonly used to study lipid metabolism is a screen for mutants with altered FA composition. Differences in FA composition can be analyzed using a gas chromatography-flame ionization detector (GC-FID) after lipids are converted to their fatty acid methyl esters. Direct transmethylation of cell pellets by acid-catalyzed transmethylation coupled with a robotized GC-FID was developed for high-throughput screening for Chlamydomonas mutants with altered FA composition (Nguyen et al. 2013). Using this method, we previously isolated a mutant defective in the $\omega-3$ FA desaturase (named $\operatorname{crfad} 7$ ) in a screen of 2,000 insertional mutants (Nguyen et al. 2013). CrFAD7 is localized to plastids and contributes to the formation of all $\omega-3$ fatty acid-containing plastidic and extraplastidic glycerolipid species (Nguyen et al. 2013).

\section{Genetic mapping of insertional mutants}

Various PCR-based techniques have been developed to identify insertion sites within the nuclear genome of Chlamydomonas, including Genome Walker, restriction enzyme sitedirected amplification-PCR (RESDA-PCR), thermal asymmetric interlaced-PCR, 3'-rapid amplification of cDNA ends, SiteFinding-PCR, and hairpin-PCR (reviewed by Li and Jonikas 2016). Among these methods, RESDA-PCR is the most commonly used and offers a high probability of identifying the insertion sites (González-Ballester et al. 2005, Kong and LiBeisson 2018). However, this approach is labor-intensive and only identifies a handful of insertion sites at once, rendering it unsuitable for the simultaneous mapping of tens of thousands of Chlamydomonas insertional mutants. Additionally, the success of these PCRbased approaches is determined largely by the PCR product size, and requires intact PCR primer binding sites in the cassette, which are occasionally disturbed by cassette truncation, concatemerization after integration, and the presence of "junk DNA" from a distant locus (Li et al. 2016).

Prompted by these challenges, a method for mapping insertion cassettes in pools of 
tens of thousands of Chlamydomonas mutants, named Mme1-based insertion site sequencing (ChlaMmeSeq), was developed (Zhang et al. 2014). Approximately $>100,000$ insertions were successfully mapped using ChlaMmeSeq (CLiP:https://www.chlamylibrary.org/). In this method, a type II restriction enzyme site (MmeI) that facilitates subsequent high-throughput sequencing is included at both ends of the transformation cassette. However, in some instances, upon fragmentation of the cassette, the binding sites for sequencing primers are eliminated, making it difficult or impossible to use ChlaMmeSeq to map that insertion site. Due to recent decreases in the cost of sequencing technologies, whole-genome re-sequencing has been used to identify point mutations and map target genes. Using this approach, we previously identified the site of genetic lesion for a Chlamydomonas mutant named constitutive-oil-accumulation 1 (coal), which was found to harbor a $>200 \mathrm{~kb}$ deletion (Goold et al. 2016).

\section{Reverse genetics in Chlamydomonas}

A successful reverse genetic approach relies on the selection of sutiable target gene(s) for genetic manipulation. There are two major ways to identify and select target genes in Chlamydomonas. First, Chlamydomonas genes that are homologous to ones already known to increase seed oil content in terrestrial plants or in other organisms can be identified. This case requires selection of the genes and rigorous confirmation of the activity of the proteins encoded by the genes. However, many genes in the Chlamydomonas database are not well annotated or characterized. In general, the Chlamydomonas genome most closely resembles those of plants, followed by those of animals and fungi. This is particularly the case for genes involved in lipid metabolism, as de novo FA synthesis occurs in the chloroplast, an organelle common to plants and algae. However, the flagellar and basal body proteins tend to show greater similarity to those of animals (Merchant et al. 2007). To select suitable target genes for reverse genetics, one can search for genes homologous to those already reported as being effective in model plant databases, such as Arabidopsis TAIR (https://www.arabidopsis.org). Further steps in selecting a target gene may include constructing phylogenetic trees; aligning the amino acid sequences of the predicted proteins; performing a subcellular localization analysis, using PredAlgo (Tardif et al. 2012); and verifying the expression profiles, using the Chlamy Network Portal (http://networks.systemsbiology.net/chlamy-portal/) or AlCOdb (http://alcodb.jp/coexpression/search). Second, candidate genes can be identified in comparative transcriptomic studies, gene co-expression analyses, or proteomics studies. 
Acyltransferases and lipid droplet-associated proteins are of particular interest to many scientists working in the field of lipid production.

\section{Strategies to improve nuclear transgene expression}

\section{Codon optimization}

Although foreign genes are easily integrated into the nuclear genome, transgene expression levels are notoriously poor in Chlamydomonas (Kong et al. 2014, Lumbreras et al. 1998, Neupert et al. 2009). The efficiency of gene expression partially depends on the nucleotide composition (particularly the GC content) of the inserted DNA fragment and on its consistency with the codon-usage of the host organism (Barahimipour et al. 2015, Neupert et al. 2009). Chlamydomonas coding sequences have an average GC content of $68 \%$ (Merchant et al. 2007). An unfavorable GC content affects gene expression at the level of chromatin structure. Recently, codon usage was reported to be more critical than GC content for gene expression efficiency in Chlamydomonas (Barahimipour et al. 2015). Codon optimization through gene re-synthesis improves transgene expression, even if Chlamydomonas contains a high GC content.

\section{Choice of promoters}

Both constitutive and inducible promoters have been developed for Chlamydomonas. Established constitutive promoters present in Chlamydomonas include the $H s p 70 A, R b c S 2$, and $P s a D$ promoters and the $H s p 70 A-R b c S 2$ chimeric promoter, and the inducible promoters include those present in the copper- $(\mathrm{Cyc} \sigma)$ and alcohol-inducible (AlcR-P alcA) promoter systems (Fischer and Rochaix 2001, Lee et al. 2018, Quinn and Merchant 1995). The AlcR$\mathrm{P}_{\mathrm{alcA}}$ system is composed of a regulatory protein (AlcR) and a promoter controlling alcA expression $\left(\mathrm{P}_{\mathrm{alcA}}\right)$, which has previously been used for inducible transgene expression in plants, and was also recently used in Chlamydomonas (Lee et al. 2018). In addition to the aforementioned traditional promoters, Scranton et al. (2016) generated a set of synthetic algal promoters (e.g., sap11) that contain a new DNA motif that is essential for promoter function and highly conserved in Chlamydomonas. The synthetic algal promoters were shown to drive higher expression of transgenes in Chlamydomonas than the strongest known endogenous promoter, the chimeric Hsp70A-RbcS2 promoter. Endogenous gene flanking sequences in Chlamydomonas have also been shown to enhance the expression level of a transgene. For 
example, a 1,425-bp upstream region of the endogenous gene $A R G 7$ can drive the expression of the $A R S 2$ reporter as strongly as the $H s p 70 A-R b c S 2$ promoter (Specht et al. 2015). LópezPaz et al. (2017) recently reported that RPL23 flanking sequences (including promoters, terminators, and UTRs) resulted in stable expression of luciferase or sh-ble, at levels much higher than $H s p 70 A-R b c S 2$ or the $P s a D$ promoter in Chlamydomonas.

\section{Synthetic intron}

Inclusion of a native intron in the cDNA of a transgene can improve expression level. For example, when the first intron of Chlamydomonas $R b c S 2(R b c S 2 i 1)$ is introduced into the coding region of ble, it dramatically increases the transformation frequency and the level of ble expression in a manner independent of orientation (Lumbreras et al. 1998). Interestingly, Eichler-Stahlberg et al. (2009) found that the addition of $R b c S 2 i l$ (e.g., one to three copies) resulted in increased expression levels of the luciferase gene in Chlamydomonas. They also showed that a particular number and genomic placement of the inserted $R b c S 2 i 1$ had an overproportionally stimulating effect on expression.

Recently, it was reported that the repetitive dispersal of $R b c S 2 i 1$ throughout the codon-optimized coding sequences (CDS) of transgenes enabled robust expression from the nuclear genome of Chlamydomonas. Using this strategy, the large sesquiterpene synthase gene $A g B s$ as well as genes encoding two farnesyl pyrophosphate synthases, ERG20 and ispA, were efficiently expressed (Lauersen et al. 2016a, Wichmann et al. 2018). The optimal $R b c S 2 i 1$ insertion site (...NG/GN...) in the codon-optimized transgene CDS for efficient splicing was also systematically determined (Baier et al. 2018). Possible reasons for the "intron-mediated efficient transgene expression" phenomenon could either be that introns contain enhancers or that introns facilitate transcript maturation and export from the nucleus; however, the mechanism remains to be elucidated.

\section{Cleavable fusion peptide system}

Nuclear transgene expression levels can be improved by incorporating resistance to a selective agent. Rasala et al. (2012) developed the ble2A system in Chlamydomonas, which enables efficient selection of transformants by transcriptional fusion of the heterologous gene to the ble selectable marker via self-cleavage of viral 2A peptides (20 amino acids). The effectiveness of this system was demonstrated by expressing various fluorescent proteins in Chlamydomonas (Rasala et al. 2013). A significantly improved ble2A system, either using an 
extended 2A peptide (39 amino acids) or combining it with an improved ultraviolet-mutant strain (UVM4), was reported to drive the high-level expression of dicistronic nuclear genes (Kong et al. 2015, Plucinak et al. 2015). However, the ability to use only one selectable marker limits the utility of the ble2A system.

Recently, Dong et al. (2017) developed the IFT25P-IFT25-2A ${ }^{\mathrm{E}}$ system, which uses the Chlamydomonas endogenous intraflagellar transport 25 (IFT25) gene instead of ble. They showed that combining a more efficient endogenous gene that is expressed at high levels (e.g., IFT25) in-frame with the 2A peptide improves transgene expression levels (Dong et al. 2017). A viral 2A peptide-based toolbox has also been used in Nannochloropsis salina (Unkefer et al. 2017). A modified 2A peptide, P2A (60 amino acids), was found to be most efficient when placed in-frame with either the ble or aph7" marker for bicistronic expression in Nannochloropsis oceanica (Poliner et al. 2018). The viral 2A peptide system is a useful tool for transgene expression in Chlamydomonas; however, the self-cleavage efficiency of the $2 \mathrm{~A}$ peptide varies based on the transgene used, and the mechanism by which the $2 \mathrm{~A}$ system drives high expression of the transgene is unclear (Rasala et al. 2013, Kong et al. 2015).

\section{Background strains}

Mutants defective in gene silencing mechanisms are useful recipients for efficient transgene expression. Several Chlamydomonas mutant lines that show improved transgene expression levels have been generated (e.g., UVM4 and UVM11) (Neupert et al. 2009). These strains have been used as background strains for protein subcellular localization studies, as host strains for protein secretion, and for the production of terpene squalene (Kajikawa et al. 2015, Lauersen et al. 2013). However, these UVM strains are still subjected to transgene silencing, probably because the gene silencing mechanisms have not been completely knocked out (Kong et al. 2015, Kurniasih et al. 2016).

We previously found that the metl mutant, which is deficient in one of the key enzymes mediating epigenetic gene silencing, showed significantly improved expression levels of nuclear transgenes, similar to UVM strains (Kong et al. 2015). When the met 1 strain was further mutated by UV irradiation, we obtained metl(UVM)-47A and B mutants, which enable robust expression of nuclear transgenes regardless of the genomic position of the insertion, due to disruption of DNA methylation-dependent and -independent silencing systems. Moreover, metl(UVM) mutants were more capable of robustly expressing native, 
fluorescent, or heterologous proteins than were other Chlamydomonas strains tested (Kurniasih et al. 2016).

\section{Tools for targeted gene repression or disruption}

In the case of essential genes, full disruption of the target gene results in cell lethality; therefore, RNAi-mediated gene repression is a more useful approach for assigning functions to these genes. RNAi approaches include expressing the antisense sequence or an inverted repeat of a gene targeted for knockdown (Schroda 2006). The Maa7/X-IR (NE-537) plasmid tool and web-based RNAi design software (e.g., THE RNAi WEB, http://www.rnaiweb.com/) facilitate the use of RNAi technology to modulate transgene expression in Chlamydomonas (Rohr et al. 2004). Despite their effectiveness, transgenes are often not stable in Chlamydomonas, and are subsequently silenced. For example, we and others have reported that RNAi silencing efficiency is reduced in Chlamydomonas after successive mitotic cell divisions (Schroda 2006, Yamasaki et al. 2008). Unstable RNAi effects, including off-target effects, limit applications of RNAi in genetic engineering of Chlamydomonas. To overcome this obstacle, a novel artificial microRNA (amiRNA) system based on ligation of DNA oligonucleotides was developed, because microRNA is normally not associated with transcriptional silencing (Molnar et al. 2009). To inactivate genes with higher accuracy and efficiency, CRISP (clustered regularly interspaced short palindromic repeats)-interference (CRISPi), which evolved from CRISPR/Cas9 (CRISPR associated nuclease 9) and uses the same type of guide RNA (gRNA), but nuclease-deficient Cas9 (Kao and Ng 2017), has recently been applied in Chlamydomonas.

Homologous recombination enables targeted gene integration, but under normal conditions, high-efficiency homologous recombination occurs only in a few algal species, such as Nannochloropsis sp. (Kilian et al. 2011). Although homologous recombination has been shown to occur during nuclear transformation in Chlamydomonas (Sodeinde and Kindle 1993), the frequency of homologous recombination is very low compared to that of random insertion (Zorin et al. 2005).

Site-specific nuclease-mediated genome editing technologies, such as the ZFN (zinc finger nuclease), TALEN (transcription activator-like effector nuclease), and CRISPR/Cas9 systems, are emerging as important alternative tools for gene knockout in microalgae. ZFN was initially used in a specially designed mutated Chlamydomonas strain, which is transformed by a non-functional aphVIII selectable marker interspaced with a short 
channelrhodopsin gene (COP3). The nonfunctional aphVIII was restored through ZFNmediated cleavage of $C O P 3$, when the cells were co-transformed with ZFNs and an aphVIII DNA template (Sizova et al. 2012). Recently, the same group optimized ZFN editing protocols for several Chlamydomonas strains (i.e., CC3403 and CC503), making ZFN-based editing a reliable and predictable tool for homologous recombination (Greiner et al. 2017). However, ZFN methodology is somewhat laborious, as a specific ZFN protein must be engineered for each target and the targeting efficiency and specificity are poor.

The TALEN system is composed of a variable number of four different DNA recognition domains, each specifically recognizing one of the four nucleotides. Gene activation induced by artificially designed TALE (dTALE) has been studied in multiple organisms including Chlamydomonas. For example, dTALE was used to target the promoters of periplasmic arylsulfatase $(A R S)$ genes in Chlamydomonas, resulting in their overexpression at both the mRNA and protein levels (Gao et al. 2014). In another study, a putative inorganic carbon transporter (HLA3), proposed to be involved in inorganic carbon uptake, was overexpressed using dTALE in Chlamydomonas (Gao et al. 2015). Furthermore, TALE in the form of TALEN was also used to induce the targeted knockout of a gene involved in a carbohydrate storage pathway (e.g., the gene encoding UDP-glucose pyrophosphorylase) in Phaeodactylum tricornutum, resulting in a strain producing higher levels of TAG (Daboussi et al. 2014).

The CRISPR-Cas9 system, consisting of two key molecules, cas9 protein and gRNA, is a faster and more accurate genome-editing tool than ZFN and TALEN. Since its discovery in 2012, the CRISPR-Cas9 system has been widely applied to land plants (Langner et al. 2018). The first trial of the CRISPR-Cas9 system in microalgae was carried out in Chlamydomonas, but resulted in a low targeting efficiency, with no surviving transformants. This is probably due to the toxicity of constitutive Cas9 expression (Jiang et al. 2014). Cytotoxicity was avoided by directly delivering Cas9 protein-gRNA ribonucleoproteins (RNPs) assembled in vitro into Chlamydomonas. However, the gene targeting efficiency (targeted colonies/preselected colonies) was still low, ranging from $0.17 \%$ to $1.4 \%$ (Shin et al. 2016). To disrupt genes in a cost-effective, routine manner, Greiner et al. (2017) optimized CRISPR/Cas9 protocols, improving the targeting efficiency (ranging from 5\% to 15\%). Using this modified method, the team disrupted eight photoreceptor genes in four different Chlamydomonas strains (Greiner et al. 2017). Deliverng another pre-assembled RNP, Lachnospiraceae Cpf1 (an ortholog of Cas9), along with single-stranded DNA repair 
templates, resulted in efficient editing and replacement of the targeted DNA at frequencies of as high as $10 \%$ in Chlamydomonas. Moreover, this method allows for transgene-free generation of sequence-specific mutations and for epitope tagging of endogenous genes (Ferenczi et al. 2017). Some examples of nuclease-directed genome editing in Chlamydomonas are provided in Table $\mathbf{1 .}$

\section{Plastidial genome engineering}

The chloroplast genome of Chlamydomonas is a primary target in efforts to produce useful recombinant proteins, predominantly because it can be readily engineered via homologous recombination and lacks transgene silencing (Rasala and Mayfield 2011). Robust heterologous protein accumulation $(\geq 5 \%$ total soluble protein) by Chlamydomonas chloroplasts was achieved by using the endogenous $p s b A$ promoter and the corresponding $5^{\prime}$ UTR to drive expression of the target gene; however, the endogenous $p s b A$ gene had to be removed, rendering the cells non-photosynthetic (Minai et al. 2006, Rasala and Mayfield 2011). To address this problem, the $16 \mathrm{~S}$ rRNA-atpA UTR hybrid promoter and synthetic regulatory elements (i.e., UTRs) have been developed to efficiently drive transgene expression in chloroplasts without having to remove enodgenous psbA (Rasala et al. 2011, Specht and Mayfield 2013). Cleavable fusion of the target gene to the C-terminal of $r b c L$ also increased foreign protein accumulation. Using this approach, the luciferase activity was increased up to 5-fold in the wild type and 33-fold in the rbcL knock-out background (Muto et al. 2009).

Modification of the $\mathrm{N}$-terminus of a transgene can also enhance foreign protein accumulation in algae. Recently, fusion of the downstream box (the first 15 codons following the start codon) of the TetC gene to the N-terminus of a transgene was shown to enhance foreign protein accumulation in Chlamydomonas chloroplasts. Its effect is probably related to translation or to other post-transcriptional processes (Richter et al. 2018). Two intercistronic expression elements from $p s b N-p s b H$ and $t s c A-c h l N$ have recently been identified (MacedoOsorio et al. 2018) that enable the expression of foreign genes (i.e., $a p h A-6$ and $g f p$ ) in Chlamydomonas chloroplasts in a synthetic operon-like manner. This approach could be useful for stacking genes for metabolic engineering or synthetic biology circuits in Chlamydomonas chloroplasts (Macedo-Osorio et al. 2018). However, chloroplasts are deficient in post-translational modifications, such as glycosylation of proteins, which limits chloroplasts as a robust platform for protein production. 
In addition to being a suitable expression platform for efficient protein production, plastids can be used to produce lipids in Chlamydomonas. For example, plastid enigineering through overexpressing plastid-localized 2-lysophosphatidic acid acyltransferase 1 (CrLPAAT1) (Yamaoka et al. 2016) can increase oil content in Chlamydomonas, as discussed below.

\section{Examples of genetic approaches for increasing oil content in Chlamydomonas}

Over the past decade, significant advances in metabolic engineering have been made to modify microalgal genomes to increase oil content. For instance, the genetic engineering tools detailed above have been used to modify lipid metabolism in Chlamydomonas through the following main approaches (Figure 1): (1) Enhancing TAG biosynthesis; (2) Increasing acetyl-CoA supply; (3) Manipulating transcription factors/regulators; (4) Blocking competing pathways; (5) Reducing lipid degradation; and (6) Modulating redox state. Some examples of engineering attempts are described below.

\section{Enhancing TAG biosynthesis}

Glycerolipids are one of the most abundant lipid classes in microalgae. Their assembly starts with the stepwise acylation of glycerol 3-phosphate (G3P) to phosphatidic acid (PA) using fatty acyl donors. The 2-lysophosphatidic acid acyltransferase (LPAAT) catalyzes the acylation at the $s n-2$ position of 2-lysophosphatidic acid to produce PA, the first common precursor of membrane and storage lipids. We reported that Chlamydomonas encodes a plastid-targeted LPAAT (CrLPAAT1) and that overexpression of CrLPAAT1 in plastids led to a more than $20 \%$ increase in oil content under nitrogen $(\mathrm{N})$-deficient conditions (Yamaoka et al. 2016). Likewise, transgenic Chlamydomonas strains overexpressing CrLPAAT2 showed greater accumulation of TAG than the parental strain (Kim et al. 2018). By contrast, RNAimediated suppression of CrLPAAT2 reduced the TAG content of the RNAi lines under N deprivation (Kim et al. 2018).

G3P dehydrogenase (GPDH) catalyzes the reversible redox conversion of dihydroxyacetone phosphate to G3P. Recently, the codon-optimized LPAAT gene from Brassica napus and GPDH1 from Saccharomyces cerevisiae were heterologously expressed in Chlamydomonas driven by the $H s p 70 A-R b c S 2$ chimeric promoter, respectively. The LPAAT and GPDH1 transgenetic lines showed a $44.5 \%$ and $67.5 \%$ increase in lipid contents, respectively, after triple heat-shock treatments (Wang et al. 2018). These results are consistent 
with those of an earlier transcriptome analysis in which GPDH and $L P A A T$ were found to be significantly upregulated when high levels of lipids accumulated in Chlamydomonas (Lv et al. 2013).

Phospholipid:diacylglycerol acyltransferase (PDAT), an acyl-CoA-independent acyltransferase, catalyzes the transfer of an acyl group from the $s n-2$ position of a phospholipid to a diacylglycerol to make TAG. This PDAT pathway is considered to be another important source of TAG biosynthesis from DAG, in addition to the acyl-CoAdependent pathway catalyzed by diacylglycerol acyltransferase (DGAT) (Li-Beisson et al. 2015). Heterologous expression of Saccharomyces cerevisiae PDAT in Chlamydomonas increased the contents of total fatty acids (FAs) and TAG by $22 \%$ and $32 \%$, respectively, under N-replete conditions (Zhu et al. 2018).

The FAs used for TAG biosynthesis can also be derived from recycled membrane lipids (i.e., monogalactosyldiacylglycerol [MGDG] and digalactosyldiacylglycerol [DGDG]). It was reported that PGD1 (PLASTID GALACTOGLYCEROLIPID DEGRADATION 1) from Chlamydomonas can hydrolyze MGDG to produce free FAs and lyso-MGDG in vitro, and the PGD1 knockout mutant had lower levels of TAG than the wild type following $\mathrm{N}$ deprivation ( $\mathrm{Li}$ et al. 2012a).

Furthermore, perturbing lipid trafficking or altering membrane lipid composition has been shown to result in TAG over-accumulation. For example, in tgd2 (trigalactosyldiacylglycerol) mutants, which are defective in lipid trafficking, excess levels of MGDG are produced that are postulated to contribute to additional acyl-groups for TAG synthesis (Warakanont et al. 2015). Additionally, the Chlamydomonas $f d x 5$ mutant, which has a defect in the ferredoxin5 gene, was shown to have changes in thylakoid membrane ultrastructure and lipid profiles in darkness (e.g., the ratio of MGDG to DGDG strongly declined), resulting in strains that hyper-accumulate TAG (Yang et al. 2015).

\section{Increasing acetyl-CoA supply}

Acetyl-CoA is an essential carbon precursor for de novo FA biosynthesis; therefore, increasing the acetyl-CoA supply by metabolic engineering could improve oil content. Phosphoenolpyruvate carboxylase (PEPC) is a key enzyme belonging to the family of decarboxylases in plants and microalgae. PEPC controls the carbon flux that enters the tricarboxylic acid (TCA) cycle, and plays an important role in carbon partitioning of substrates in competition with lipid synthesis (Deng et al. 2014, Izui et al. 2004). In 
Chlamydomonas, inhibiting PEPC1 activity by RNAi or CRISPRi-based techniques increased the oil content by $20 \%$, probably by enhancing the flow of carbon to FA synthesis (Deng et al. 2014, Kao and Ng 2017). Down-regulating both PEPC isoforms (CrPEPC1 and CrPEPC2) by amiRNA resulted in a $29 \%$ to $48 \%$ increase in total FA levels, respectively, which was suggested to occur due to the flux of carbon away from the TCA cycle, and the subsequent increase in the availability of acetyl-CoA for FA biosynthesis (Wang et al. 2017). Consistent with this hypothesis, the expression levels of TAG biosynthesis-related genes, such as DGAT2 and phosphatidate phosphatase (PAP2), were increased in CrPEPC1 RNAi strains (Deng et al. 2014).

\section{Manipulating transcription factors/regulators}

Manipulating the expression of transcription factor (TF) genes usually affects multiple steps/genes of the same pathway, thereby upregulating the entire pathway rather than a specific enzymatic step. TF engineering is a promising strategy, because it can circumvent the secondary bottlenecks commonly observed when only a single gene is targeted (Courchesne et al. 2009). TFs showing positive or negative correlations with lipid biosynthetic genes at the RNA expression level could be potential targets for engineering of oil content (Hu et al. 2014). Dof-binding sequences are abundant in the promoter regions of many genes related to lipid biosynthesis (Wang et al. 2006), indicating that Dof genes might contribute to lipid biosynthesis by upregulating genes associated with FA biosynthesis. Indeed, a study in higher plants showed that overexpression of Dof-type TF genes (GmDofl and GmDof14) from soybean (Glycine max) enhanced TAG accumulation in the seeds of transgenic Arabidopsis plants (Wang et al. 2007). A codon-optimized soybean DOF-type TF gene (GenBank ID: DQ857261.1) was subsequently heterologously expressed in Chlamydomonas, and the resulting transgenic lines had an up to 2-fold increase in lipid content (Ibáñez-Salazar et al. 2014). Ngan et al. (2015) identified another TF named PSR1 (PHOSPHORUS STRESS RESPONSE 1), which triggers cytosolic lipid accumulation, as a pivotal switch in carbon storage metabolism in Chlamydomonas. Overexpression of PSRI increased TAG accumulation 2-fold (on a dry weight basis) in cells grown under normal culture conditions without growth inhibition (Ngan et al. 2015).

We recently isolated a Chlamydomonas mutant (starch degradation 1, std1) deficient in a dual-specificity tyrosinephosphorylation-regulated kinase (DYRK) (Schulz-Raffelt et al. 2016). The stdl mutant accumulates higher levels of oil than the wild type under 
photoautotrophic N starvation conditions. By contrast, another DYRK, named TAR1 (TAG ACCUMULATION REGULATOR 1), was found to positively regulate TAG accumulation under mixotrophic $\mathrm{N}$ and $\mathrm{S}$ deficiency, and the tar1-1 mutant accumulated lower levels of TAG than did the wild-type strain (Kajikawa et al. 2015b). The effect on oil content has not yet been evaluated for tarl under photoautotrophic conditions. This plant-specific DYRK kinase (DYRKP), which has been proposed to act as a positive or negative regulator of sink capacity and thereby integrate nutrient and energy signals (Schulz-Raffelt et al. 2016), represents a promising target for improving energy density in microalgae.

Additionally, VIP1, an inositol hexakisphosphate/diphosphoinositol phosphate kinase involved in phosphatidylinositol signaling, has recently been reported to show synergism with target of rapamycin kinase signaling, which governs carbon metabolism and lipid accumulation in Chlamydomonas. The null vip1-1 mutant exhibits increased levels of TAG and a higher content of lipid droplets in both N-replete and -deplete conditions (Couso et al. 2016). A comprehensive understanding of the underlying mechanism by which TFs regulate lipid alteration and the discovery of TFs regulating algal lipid metabolism will facilitate targeted engineering strategies to improve lipid production in microalgae.

\section{Blocking competing pathways}

Disrupting competing metabolic pathways of lipid biosynthesis has been routinely used to enhance lipid productivity. In addition to TAG, starch is another primary carbon sink in green microalgae. Previous work showed that knocking-out starch synthesis could increase oil content ( $\mathrm{Li}$ et al. 2010, Work et al. 2010); however, this does not always seem to be the case (Siaut et al. 2011, Vonlanthen et al. 2015), and varied depending on the background strains and cultivation conditions. The relationship between starch synthesis and TAG accumulation merits further investigation.

\section{Reducing lipid degradation}

Cellular lipid content is the end result of an equilibrium between synthesis and degradation. Therefore, lipid hydrolytic enzymes (i.e., lipases) are also potential targets for engineering efforts to increase TAG content. In Chlamydomonas, the lipase CrLIP1 was found to be a DAG lipase with broad substrate specificity. Silencing of CrLIP1 resulted in an increase in oil content following $\mathrm{N}$ replenishment upon $\mathrm{N}$ starvation due to the arrest of TAG hydrolysis (Li et al. 2012). TAG degradation is also directly linked to the cell cycle in Chlamydomonas. The 
insertional null mutant cht7 (compromised hydrolysis of triacylglycerols 7) is severely impaired in TAG degradation, and the TAG level in this mutant remains 10-fold higher than that of wild-type cells upon $\mathrm{N}$ resupply after $\mathrm{N}$ starvation, due to a defect in exiting quiescence (Tsai et al. 2014).

Recently, we reported that functional FA $\beta$-oxidation is involved in lipid homeostasis during nutrient stress in Chlamydomonas. In the insertional knockout mutants for ACX2 (ACYL-COA OXIDASE 2), a key enzyme in peroxisomal FA $\beta$-oxidation, oil remobilization is severely blocked (by 60-80\%) upon $\mathrm{N}$ resupply after $\mathrm{N}$ starvation and oil content is increased by $20 \%$ during $\mathrm{N}$ starvation (Kong et al. 2017). In Chlamydomonas, with the exception of isocitrate lyase (ICL), all other enzymes of the glyoxylate cycle are localized in peroxisomes (Lauersen et al. 2016b). The null icl mutant overaccumulated total FAs when cultivated mixotrophically, presumably due to a reduction in the $\beta$-oxidation of FAs (Plancke et al. 2014). Overall, reduced lipid turnover may enhance the net oil content; therefore, identifying and engineering genes involved in lipid degradation is a promising avenue toward increasing oil content in microalgae.

\section{Modulating redox state}

The FA synthase complex is activated by NADPH (Rawsthorne 2002), and a positive link between the level of NADPH and FA content has been established in microalgae (Osada et al. 2017, Xue et al. 2015). Malic enzyme (ME) catalyzes the conversion of malate to pyruvate and simultaneously reduces $\mathrm{NADP}^{+}$to NADPH. The total lipid content and the neutral lipid content were increased 2.5-fold and 3.2-fold via transgenic overexpression of PtME in Phaeodactylum tricornutum and Chlorella pyrenoidosa, respectively (Xue et al. 2015, 2016). Recently, the enzyme glucose-6-phosphate dehydrogenase (G6PD), which is involved in the formation of NADPH in the pentose phosphate pathway, was reported to contribute to lipid synthesis in the oleaginous diatoms Phaeodactylum tricornutum and Fistulifera solaris. Overexpression of G6PD elevated lipid productivity 2.7-fold in Phaeodactylum tricornutum during the stationary phase, and 1.5-fold in Fistulifera under N depletion (Osada et al. 2017, Xue et al. 2017).

Malate dehydrogenase $(\mathrm{MDH})$ catalyzes the reversible conversion of $\mathrm{NAD}(\mathrm{P}) \mathrm{H}$ to $\mathrm{NAD}(\mathrm{P})^{+}$via the reduction of oxaloacetate to malate (Mettler and Beevers 1980). Recently, we isolated and characterized a Chlamydomonas mutant deficient in the peroxisomal $\mathrm{NAD}^{+}-$ dependent $M D H 2$. In its absence, $m d h 2$ mutant cells are compromised in TAG breakdown and 
showed an increased capacity to make $\mathrm{NAD}(\mathrm{P}) \mathrm{H}$, resulting in a $>50 \%$ increase in storage lipids and a 200\% increase in starch during photoautotrophic $\mathrm{N}$ deprivation (Kong et al. 2018b). Enhancing the NADPH supply using metabolic engineering techniques seems to be a promising strategy for boosting lipid accumulation in microalgae.

\section{Conclusion and prospectives}

Here we reviewed the essential techniques for improving the oil content of Chlamydomonas, from transformation methodologies to genome engineering strategies. The state-of-the-art CRISPR-Cas9 system shows great potential for precise genetic engineering, and has been successfully used in Chlamydomonas, diatoms, and Nannochloropsis. The continued progress of synthetic biology in Chlamydomonas will facilitate the development of the microalgal biotechnology sector.

We also described cases in which genetic tools were succesfully used to increase oil content in Chlamydomonas. It is apparent that microalgae that accumulate oils have acquired not only divergent enzymes that produce these FAs, but also a large number of enzymes (e.g., acyltransferases) that can effectively channel these FAs into oil. Understanding and manipulating the regulatory processes of lipid metabolism may turn out to be even more effective than manipulating specific enzymes. Thus, boosting oil accumulation in microalgae may require a combination of strategies involving both genetic engineering tools and synthetic biology approaches employing many genes. A breakthrough in this technology is hopefully not far off.

\section{Disclosures}

The authors have no conflicts of interest to declare.

\section{Acknowledgements}

This work was supported by the Advanced Biomass R\&D Center (ABC) of Global Frontier Project funded by the Ministry of Science, ICT and Future Planning (ABC2015M3A6A2065746) and a grant from the BioGreen 21 Program (No. PJ013412), Rural Development Administration, Republic of Korea awarded to Y. Lee, and the Korea Research Fellowship Program funded by the Ministry of Science, ICT and Future Planning through the National Research Foundation of Korea (2016H1D3A1909463) to Y. Yamaoka. The work was also supported by the Start-up Fund of Introduction of Talent Program awarded to F. 
Kong, and French Agence Nationale pour la Recherche (ANR-MUsCA) awarded to Y. LiBeisson.

\section{References}

Baek, K., Kim, D.H., Jeong, J., Sim, S.J., Melis, A., Kim, J.-S., et al. (2016) DNA-free twogene knockout in Chlamydomonas reinhardtii via CRISPR-Cas9 ribonucleoproteins. Sci. Rep. 6: 30620.

Baier, T., Wichmann, J., Kruse, O., and Lauersen, K.J. (2018). Intron-containing algal transgenes mediate efficient recombinant gene expression in the green microalga Chlamydomonas reinhardtii. Nucleic Acids Res. 46: 6909-6919.

Barahimipour, R., Strenkert, D., Neupert, J., Schroda, M., Merchant, S.S., and Bock, R. (2015) Dissecting the contributions of GC content and codon usage to gene expression in the model alga Chlamydomonas reinhardtii. Plant J. 84: 704-717.

Boyle, N.R., Page, M.D., Liu, B., Blaby, I.K., Casero, D., Kropat, J., et al. (2012) Three acyltransferases and nitrogen-responsive regulator are implicated in nitrogen starvationinduced triacylglycerol accumulation in Chlamydomonas. J. Biol. Chem. 287: 1581115825 .

Cagnon, C., Mirabella, B., Nguyen, H.M., Beyly-Adriano, A., Bouvet, S., Cuiné, S., et al. (2013) Development of a forward genetic screen to isolate oil mutants in the green microalga Chlamydomonas reinhardtii. Biotechnol. Biofuels. 6: 178.

Cheng, X., Liu, G., Ke, W., Zhao, L., Lv, B., Ma, X., et al. (2017) Building a multipurpose insertional mutant library for forward and reverse genetics in Chlamydomonas. Plant Methods. 13: 36.

Chew, K.W., Yap, J.Y., Show, P.L., Suan, N.H., Juan, J.C., Ling, T.C., et al. (2017) Microalgae biorefinery: High value products perspectives. Bioresour. Technol. 229: 53-62.

Courchesne, N.M.D., Parisien, A., Wang, B., and Lan, C.Q. (2009) Enhancement of lipid production using biochemical, genetic and transcription factor engineering approaches. $J$. Biotechnol. 141: 31-41.

Couso, I., Evans, B., Li, J., Liu, Y., Ma, F., Diamond, S., et al. (2016) Synergism between inositol polyphosphates and TOR kinase signaling in nutrient sensing, growth control and lipid metabolism in Chlamydomonas. Plant Cell. 28: 2026-2042.

Crozet, P., Navarro, F.J., Willmund, F., Mehrshahi, P., Bakowski, K., Lauersen, K.J., et al. (2018) Birth of a photosynthetic chassis: a MoClo toolkit enabling synthetic biology in the microalga Chlamydomonas reinhardtii. ACS Synth. Biol. 21: 2074-2086

Daboussi, F., Leduc, S., Maréchal, A., Dubois, G., Guyot, V., Perez-Michaut, C., et al. (2014) Genome engineering empowers the diatom Phaeodactylum tricornutum for biotechnology. Nat. Commun. 5: 3831. 
Deng, X., Li, Y., and Fei, X. (2011) The mRNA abundance of pepc2 gene is negatively correlated with oil content in Chlamydomonas reinhardtii. Biomass Bioenergy. 35: 18111817.

Deng, X., Cai, J., Li, Y., and Fei, X. (2014) Expression and knockdown of the PEPC1 gene affect carbon flux in the biosynthesis of triacylglycerols by the green alga Chlamydomonas reinhardtii. Biotechnol. Lett. 36: 2199-2208.

Dent, R.M., Sharifi, M.N., Malnoë, A., Haglund, C., Calderon, R.H., Wakao, S., et al. (2015) Large-scale insertional mutagenesis of Chlamydomonas supports phylogenomic functional prediction of photosynthetic genes and analysis of classical acetate-requiring mutants. Plant J. 82: 337-351.

Dong, B., Hu, H.-H., Li, Z.-F., Cheng, R.-Q., Meng, D.-M., Wang, J., et al. (2017) A novel bicistronic expression system composed of the intraflagellar transport protein gene ift 25 and FMDV 2A sequence directs robust nuclear gene expression in Chlamydomonas reinhardtii. Appl. Microbiol. Biotechnol. 101: 4227-4245.

Du, Z.-Y., and Benning, C. (2016) Triacylglycerol Accumulation in Photosynthetic Cells in Plants and Algae. In Lipids in Plant and Algae Development. Edited by Nakamura Y., LiBeisson Y. pp. 179-205. Springer, Cham.

Eichler-Stahlberg, A., Weisheit, W., Ruecker, O., and Heitzer, M. (2009) Strategies to facilitate transgene expression in Chlamydomonas reinhardtii. Planta. 229: 873-883.

Ferenczi, A., Pyott, D.E., Xipnitou, A., and Molnar, A. (2017) Efficient targeted DNA editing and replacement in Chlamydomonas reinhardtii using Cpf1 ribonucleoproteins and singlestranded DNA. Proc. Natl. Acad. Sci. 114: 13567-13572.

Fischer, N., and Rochaix, J.D. (2001) The flanking regions of PsaD drive efficient gene expression in the nucleus of the green alga Chlamydomonas reinhardtii. Mol. Genet. Genomics. 265: 888-894.

Fuhrmann, M., Oertel, W., and Hegemann, P. (1999) A synthetic gene coding for the green fluorescent protein (GFP) is a versatile reporter in Chlamydomonas reinhardtii. Plant J. 19: 353-361.

Gao, H., Wright, D.A., Li, T., Wang, Y., Horken, K., Weeks, D.P., et al. (2014) TALE activation of endogenous genes in Chlamydomonas reinhardtii. Algal Res. 5: 52-60.

Gao, H., Wang, Y., Fei, X., Wright, D.A., and Spalding, M.H. (2015) Expression activation and functional analysis of HLA3, a putative inorganic carbon transporter in Chlamydomonas reinhardtii. Plant J. 82: 1-11.

Goold, H.D., Nguyen, H.M., Kong, F., Beyly-Adriano, A., Légeret, B., Billon, E., et al. (2016) Whole Genome Re-Sequencing Identifies a Quantitative Trait Locus Repressing Carbon Reserve Accumulation during Optimal Growth in Chlamydomonas reinhardtii. Sci. Rep. 6: 25209 
Greiner, A., Kelterborn, S., Evers, H., Kreimer, G., Sizova, I., and Hegemann, P. (2017) Targeting of Photoreceptor Genes in Chlamydomonas reinhardtii via Zinc-Finger Nucleases and CRISPR/Cas9. Plant Cell. 29: 2498-2518.

González-Ballester, D., de Montaigu, A., Galván, A., and Fernández, E. (2005) Restriction enzyme site-directed amplification PCR: a tool to identify regions flanking a marker DNA. Anal. Biochem. 340: 330-335.

Hou, L., Wang, P., Kong, F., Park, H., Kobiro, K., and Ohama, T. (2013) Mesoporous TiO2 nanoparticles: A new material for biolistic bombardment. Phycol. Res. 61: 58-60.

Hu, J., Wang, D., Li, J., Jing, G., Ning, K., and Xu, J. (2014) Genome-wide identification of transcription factors and transcription-factor binding sites in oleaginous microalgae Nannochloropsis. Sci. Rep. 4: 5454.

Hu, Q., Sommerfeld, M., Jarvis, E., Ghirardi, M., Posewitz, M., Seibert, M., et al. (2008) Microalgal triacylglycerols as feedstocks for biofuel production: Perspectives and advances. Plant J. 54: 621-639.

Ibáñez-Salazar, A., Rosales-Mendoza, S., Rocha-Uribe, A., Ramírez-Alonso, J.I., LaraHernández, I., Hernández-Torres, A., et al. (2014) Over-expression of Dof-type transcription factor increases lipid production in Chlamydomonas reinhardtii. $J$. Biotechnol. 184: 27-38.

Izui, K., Matsumura, H., Furumoto, T., and Kai, Y. (2004) Phosphoenolpyruvate carboxylase: a new era of structural biology. Annu. Rev. Plant Biol. 55: 69-84.

Jeong, J., Baek, K., Yu, J., Kirst, H., Betterle, N., Shin, W., et al. (2018) Deletion of the chloroplast LTD protein impedes LHCI import and PSI-LHCI assembly in Chlamydomonas reinhardtii. J. Exp. Bot. 69: 1147-1158.

Jiang, W., Brueggeman, A.J., Horken, K.M., Plucinak, T.M., and Weeks, D.P. (2014) Successful transient expression of Cas9 and single guide RNA genes in Chlamydomonas reinhardtii. Eukaryot. Cell. 13: 1465-1469.

Jiang, W.Z., and Weeks, D.P. (2017) A gene-within-a-gene Cas9/sgRNA hybrid construct enables gene editing and gene replacement strategies in Chlamydomonas reinhardtii. Algal Res. 26: 474-480.

Jinkerson, R.E., and Jonikas, M.C. (2015) Molecular techniques to interrogate and edit the Chlamydomonas nuclear genome. Plant J. 82: 393-412.

Kajikawa, M., Kinohira, S., Ando, A., Shimoyama, M., Kato, M., and Fukuzawa, H. (2015a) Accumulation of squalene in a microalga Chlamydomonas reinhardtii by genetic modification of squalene synthase and squalene epoxidase genes. PloS One. 10: e0120446.

Kajikawa, M., Sawaragi, Y., Shinkawa, H., Yamano, T., Ando, A., Kato, M., et al. (2015b) Algal dual-specificity tyrosine phosphorylation-regulated kinase, triacylglycerol accumulation regulator1, regulates accumulation of triacylglycerol in nitrogen or sulfur deficiency. Plant Physiol. 168: 752-764. 
Kao, P.-H., and Ng, I.-S. (2017) CRISPRi mediated phosphoenolpyruvate carboxylase regulation to enhance the production of lipid in Chlamydomonas reinhardtii. Bioresour. Technol. 245: 1527-1537.

Kilian, O., Benemann, C.S.E., Niyogi, K.K., and Vick, B. (2011) High-efficiency homologous recombination in the oil-producing alga Nannochloropsis sp. Proc. Natl. Acad. Sci. 108: $21265-21269$.

Kim, Y., Terng, E.L., Riekhof, W.R., Cahoon, E.B., and Cerutti, H. (2018) Endoplasmic reticulum acyltransferase with prokaryotic substrate preference contributes to triacylglycerol assembly in Chlamydomonas. Proc. Natl. Acad. Sci. U. S. A. 115: 16521657.

Kong, F., and Li-Beisson, Y. (2018) Identification of Insertion Site by RESDA-PCR in Chlamydomonas Mutants Generated by AphVIII Random Insertional Mutagenesis. BIOProtoc. 8: e2718.

Kong, F., Yamasaki, T., and Ohama, T. (2014) Expression levels of domestic cDNA cassettes integrated in the nuclear genomes of various Chlamydomonas reinhardtii strains. J. Biosci. Bioeng. 117: 613-616.

Kong, F., Yamasaki, T., Kurniasih, S.D., Hou, L., Li, X., Ivanova, N., et al. (2015) Robust expression of heterologous genes by selection marker fusion system in improved Chlamydomonas strains. J. Biosci. Bioeng. 120: 239-245.

Kong, F., Liang, Y., Légeret, B., Beyly-Adriano, A., Blangy, S., Haslam, R.P., et al. (2017) Chlamydomonas carries out fatty acid $\beta$-oxidation in ancestral peroxisomes using a bona fide acyl-CoA oxidase. Plant J. 90: 358-371.

Kong, F., Romero, I.T., Warakanont, J., and Li-Beisson, Y. (2018a) Lipid catabolism in microalgae. New Phytol. 218: 1340-1348.

Kong, F., Burlacot, A., Liang, Y., Légeret, B., Alseekh, S., Brotman, Y., et al. (2018b) Interorganelle Communication: Peroxisomal MALATE DEHYDROGENASE2 Connects Lipid Catabolism to Photosynthesis through Redox Coupling in Chlamydomonas. Plant Cell. 30: 1824-1847.

Kurniasih, S.D., Yamasaki, T., Kong, F., Okada, S., Widyaningrum, D., and Ohama, T. (2016) UV-mediated Chlamydomonas mutants with enhanced nuclear transgene expression by disruption of DNA methylation-dependent and independent silencing systems. Plant Mol. Biol. 92: 629-641.

Langner, T., Kamoun, S., and Belhaj, K. (2018) CRISPR Crops: Plant Genome Editing Toward Disease Resistance. Annu. Rev. Phytopathol. 56: 479-512.

Lauersen, K.J., Berger, H., Mussgnug, J.H., and Kruse, O. (2013) Efficient recombinant protein production and secretion from nuclear transgenes in Chlamydomonas reinhardtii. $J$. Biotechnol. 167: 101-110. 
Lauersen, K.J., Baier, T., Wichmann, J., Wördenweber, R., Mussgnug, J.H., Hübner, W., et al. (2016a) Efficient phototrophic production of a high-value sesquiterpenoid from the eukaryotic microalga Chlamydomonas reinhardtii. Metab. Eng. 38: 331-343.

Lauersen, K.J., Willamme, R., Coosemans, N., Joris, M., Kruse, O., and Remacle, C. (2016b) Peroxisomal microbodies are at the crossroads of acetate assimilation in the green microalga Chlamydomonas reinhardtii. Algal Res. 16: 266-274.

Lee, B., Choi, G.-G., Choi, Y.-E., Sung, M., Park, M.S., and Yang, J.-W. (2014) Enhancement of lipid productivity by ethyl methane sulfonate-mediated random mutagenesis and proteomic analysis in Chlamydomonas reinhardtii. Korean J. Chem. Eng. 31: $1036-1042$.

Lee, S., Lee, Y.J., Choi, S., Park, S.-B., Tran, Q.-G., Heo, J., et al. (2018) Development of an alcohol-inducible gene expression system for recombinant protein expression in Chlamydomonas reinhardtii. J. Appl. Phycol. 30: 2297-2304.

Li, X., and Jonikas, M.C. (2016) High-Throughput Genetics Strategies for Identifying New Components of Lipid Metabolism in the Green Alga Chlamydomonas reinhardtii. In Lipids in Plant and Algae Development. Edited by Nakamura Y., Li-Beisson Y. pp. 223-247. Springer, Cham.

Li, X., Moellering, E.R., Liu, B., Johnny, C., Fedewa, M., Sears, B.B., et al. (2012a). A galactoglycerolipid lipase is required for triacylglycerol accumulation and survival following nitrogen deprivation in Chlamydomonas reinhardtii. Plant Cell. 24: 4670-4686.

Li, X., Benning, C., and Kuo, M.-H. (2012b) Rapid Triacylglycerol Turnover in Chlamydomonas reinhardtii Requires a Lipase with Broad Substrate Specificity. Eukaryot. Cell. 11: 1451-1462.

Li, X., Zhang, R., Patena, W., Gang, S.S., Blum, S.R., Ivanova, N., et al. (2016) An Indexed, Mapped Mutant Library Enables Reverse Genetics Studies of Biological Processes in Chlamydomonas reinhardtii. Plant Cell. 28: 367-387.

Li, Y., Han, D., Hu, G., Dauvillee, D., Sommerfeld, M., Ball, S., et al. (2010) Chlamydomonas starchless mutant defective in ADP-glucose pyrophosphorylase hyperaccumulates triacylglycerol. Metab. Eng. 12: 387-391.

Li-Beisson, Y., Beisson, F., and Riekhof, W. (2015) Metabolism of acyl-lipids in Chlamydomonas reinhardtii. Plant J. 82: 504-522.

Lim, J.-M., Ahn, J.-W., Hwangbo, K., Choi, D.-W., Park, E.-J., Hwang, M.S., et al. (2013) Development of cyan fluorescent protein (CFP) reporter system in green alga Chlamydomonas reinhardtii and macroalgae Pyropia sp. Plant Biotechnol. Rep. 7: 407414.

Lin, G., Wang, Y., Guo, L., Ding, H., Hu, Y., Liang, S., et al. (2017) Verification of mutagen function of Zeocin in Nannochloropsis oceanica through transcriptome analysis. J. Ocean Univ. China. 16: 501-508. 
López-Paz, C., Liu, D., Geng, S., and Umen, J.G. (2017) Identification of Chlamydomonas reinhardtii endogenous genic flanking sequences for improved transgene expression. Plant J. Cell Mol. Biol. 92: 1232-1244.

Lumbreras, V., Stevens, D.R., and Purton, S. (1998) Efficient foreign gene expression in Chlamydomonas reinhardtii mediated by an endogenous intron. Plant J. 14: 441-447.

Lv, H., Qu, G., Qi, X., Lu, L., Tian, C., and Ma, Y. (2013) Transcriptome analysis of Chlamydomonas reinhardtii during the process of lipid accumulation. Genomics. 101: 229-237.

Macedo-Osorio, K.S., Pérez-España, V.H., Garibay-Orijel, C., Guzmán-Zapata, D., DuránFigueroa, N.V., and Badillo-Corona, J.A. (2018) Intercistronic expression elements (IEE) from the chloroplast of Chlamydomonas reinhardtii can be used for the expression of foreign genes in synthetic operons. Plant Mol. Biol. doi: 10.1007/s11103-018-0776-z

Merchant, S.S., Prochnik, S.E., Vallon, O., Harris, E.H., Karpowicz, S.J., Witman, G.B., et al. (2007) The Chlamydomonas Genome Reveals the Evolution of Key Animal and Plant Functions. Science. 318: 245-250.

Merchant, S.S., Kropat, J., Liu, B., Shaw, J., and Warakanont, J. (2012) TAG, You're it! Chlamydomonas as a reference organism for understanding algal triacylglycerol accumulation. Curr. Opin. Biotechnol. 23: 352-363.

Meslet-Cladière, L., and Vallon, O. (2011) Novel Shuttle Markers for Nuclear Transformation of the Green Alga Chlamydomonas reinhardtii. Eukaryot. Cell. 10: 1670-1678.

Mettler, I.J., and Beevers, H. (1980) Oxidation of NADH in Glyoxysomes by a MalateAspartate Shuttle. Plant Physiol. 66: 555-560.

Minai, L., Wostrikoff, K., Wollman, F.-A., and Choquet, Y. (2006) Chloroplast biogenesis of photosystem II cores involves a series of assembly-controlled steps that regulate translation. Plant Cell. 18: 159-175.

Mini, P., Demurtas, O.C., Valentini, S., Pallara, P., Aprea, G., Ferrante, P., et al. (2018) Agrobacterium-mediated and electroporation-mediated transformation of Chlamydomonas reinhardtii: a comparative study. BMC Biotechnol. 18: 11.

Molnar, A., Bassett, A., Thuenemann, E., Schwach, F., Karkare, S., Ossowski, S., et al. (2009) Highly specific gene silencing by artificial microRNAs in the unicellular alga Chlamydomonas reinhardtii. Plant J. 58: 165-174.

Moellering, E.R., and Benning, C. (2010) RNA interference silencing of a major lipid droplet protein affects lipid droplet size in Chlamydomonas reinhardtii. Eukaryot. Cell. 9: 97-106.

Muto, M., Henry, R.E., and Mayfield, S.P. (2009) Accumulation and processing of a recombinant protein designed as a cleavable fusion to the endogenous Rubisco LSU protein in Chlamydomonas chloroplast. BMC Biotechnol. 9: 26.

Neupert, J., Karcher, D., and Bock, R. (2009) Generation of Chlamydomonas strains that efficiently express nuclear transgenes. Plant J. 57: 1140-1150. 
Ngan, C.Y., Wong, C.-H., Choi, C., Yoshinaga, Y., Louie, K., Jia, J., et al. (2015) Lineagespecific chromatin signatures reveal a regulator of lipid metabolism in microalgae. Nat. Plants. 1: 15107.

Nguyen, H.M., Cuiné, S., Beyly-Adriano, A., Légeret, B., Billon, E., Auroy, P., et al. (2013) The green microalga Chlamydomonas reinhardtii has a single $\omega-3$ fatty acid desaturase that localizes to the chloroplast and impacts both plastidic and extraplastidic membrane lipids. Plant Physiol. 163: 914-928.

Osada, K., Maeda, Y., Yoshino, T., Nojima, D., Bowler, C., and Tanaka, T. (2017) Enhanced NADPH production in the pentose phosphate pathway accelerates lipid accumulation in the oleaginous diatom Fistulifera solaris. Algal Res. 23: 126-134.

Plancke, C., Vigeolas, H., Höhner, R., Roberty, S., Emonds-Alt, B., Larosa, V., et al. (2014) Lack of isocitrate lyase in Chlamydomonas leads to changes in carbon metabolism and in the response to oxidative stress under mixotrophic growth. Plant J. 77: 404-417.

Plucinak, T.M., Horken, K.M., Jiang, W., Fostvedt, J., Nguyen, S.T., and Weeks, D.P. (2015) Improved and versatile viral $2 \mathrm{~A}$ platforms for dependable and inducible high-level expression of dicistronic nuclear genes in Chlamydomonas reinhardtii. Plant J. 82: 717729.

Poliner, E., Pulman, J.A., Zienkiewicz, K., Childs, K., Benning, C., and Farré, E.M. (2018) A toolkit for Nannochloropsis oceanica CCMP1779 enables gene stacking and genetic engineering of the eicosapentaenoic acid pathway for enhanced long-chain polyunsaturated fatty acid production. Plant Biotechnol. J. 16: 298-309.

Quinn, J.M., and Merchant, S. (1995) Two copper-responsive elements associated with the Chlamydomonas Cyc6 gene function as targets for transcriptional activators. Plant Cell. 7: $623-628$.

Rasala, B.A., and Mayfield, S.P. (2011) The microalga Chlamydomonas reinhardtii as a platform for the production of human protein therapeutics. Bioeng. Bugs. 2: 50-54.

Rasala, B.A., Muto, M., Sullivan, J., and Mayfield, S.P. (2011) Improved heterologous protein expression in the chloroplast of Chlamydomonas reinhardtii through promoter and 5' untranslated region optimization. Plant Biotechnol. J. 9: 674-683.

Rasala, B.A., Lee, P.A., Shen, Z., Briggs, S.P., Mendez, M., and Mayfield, S.P. (2012) Robust expression and secretion of Xylanasel in Chlamydomonas reinhardtii by fusion to a selection gene and processing with the FMDV 2A peptide. PloS One. 7: e43349.

Rasala, B.A., Barrera, D.J., Ng, J., Plucinak, T.M., Rosenberg, J.N., Weeks, D.P., et al. (2013) Expanding the spectral palette of fluorescent proteins for the green microalga Chlamydomonas reinhardtii. Plant J. 74: 545-556.

Rawsthorne, S. (2002) Carbon flux and fatty acid synthesis in plants. Prog. Lipid Res. 41: 182-196. 
Richter, L.V., Yang, H., Yazdani, M., Hanson, M.R., and Ahner, B.A. (2018) A downstream box fusion allows stable accumulation of a bacterial cellulase in Chlamydomonas reinhardtii chloroplasts. Biotechnol. Biofuels. 11: 133.

Rohr, J., Sarkar, N., Balenger, S., Jeong, B., and Cerutti, H. (2004) Tandem inverted repeat system for selection of effective transgenic RNAi strains in Chlamydomonas. Plant J. 40: 611-621.

Schroda, M. (2006) RNA silencing in Chlamydomonas: mechanisms and tools. Curr. Genet. 49: 69-84.

Schulz-Raffelt, M., Chochois, V., Auroy, P., Cuiné, S., Billon, E., Dauvillée, D., et al. (2016) Hyper-accumulation of starch and oil in a Chlamydomonas mutant affected in a plantspecific DYRK kinase. Biotechnol. Biofuels. 9: 55.

Scranton, M.A., Ostrand, J.T., Georgianna, D.R., Lofgren, S.M., Li, D., Ellis, R.C., et al. (2016) Synthetic promoters capable of driving robust nuclear gene expression in the green alga Chlamydomonas reinhardtii. Algal Res. 15: 135-142.

Shimogawara, K., Fujiwara, S., Grossman, A., and Usuda, H. (1998) High-efficiency transformation of Chlamydomonas reinhardtii by electroporation. Genetics. 148: 18211828 .

Shin, S.-E., Lim, J.-M., Koh, H.G., Kim, E.K., Kang, N.K., Jeon, S., et al. (2016) CRISPR/Cas9-induced knockout and knock-in mutations in Chlamydomonas reinhardtii. Sci. Rep. 6: 27810.

Shin, Y.S., Jeong, J., Nguyen, T.H.T., Kim, J.Y.H., Jin, E., and Sim, S.J. (2019) Targeted knockout of phospholipase A2 to increase lipid productivity in Chlamydomonas reinhardtii for biodiesel production. Bioresour. Technol. 271: 368-374.

Siaut, M., Cuiné, S., Cagnon, C., Fessler, B., Nguyen, M., Carrier, P., et al. (2011) Oil accumulation in the model green alga Chlamydomonas reinhardtii: characterization, variability between common laboratory strains and relationship with starch reserves. $B M C$ Biotechnol. 11: 7 .

Sizova, I., Greiner, A., Awasthi, M., Kateriya, S., and Hegemann, P. (2012) Nuclear gene targeting in Chlamydomonas using engineered zinc-finger nucleases. Plant J. 73: 873-882.

Sodeinde, O.A., and Kindle, K.L. (1993) Homologous recombination in the nuclear genome of Chlamydomonas reinhardtii. Proc. Natl. Acad. Sci. U. S. A. 90: 9199-9203.

Specht, E.A., and Mayfield, S.P. (2013) Synthetic oligonucleotide libraries reveal novel regulatory elements in Chlamydomonas chloroplast mRNAs. ACS Synth. Biol. 2: 34-46.

Specht, E.A., Nour-Eldin, H.H., Hoang, K.T.D., and Mayfield, S.P. (2015) An improved ARS2-derived nuclear reporter enhances the efficiency and ease of genetic engineering in Chlamydomonas. Biotechnol. J. 10: 473-479.

Tardif, M., Atteia, A., Specht, M., Cogne, G., Rolland, N., Brugière, S., et al. (2012) PredAlgo: A New Subcellular Localization Prediction Tool Dedicated to Green Algae. Mol. Biol. Evol. 29: 3625-3639. 
Terashima, M., Freeman, E.S., Jinkerson, R.E., and Jonikas, M.C. (2015) A fluorescenceactivated cell sorting-based strategy for rapid isolation of high-lipid Chlamydomonas mutants. Plant J. 81: 147-159.

Tsai, C.-H., Warakanont, J., Takeuchi, T., Sears, B.B., Moellering, E.R., and Benning, C. (2014) The protein Compromised Hydrolysis of Triacylglycerols 7 (CHT7) acts as a repressor of cellular quiescence in Chlamydomonas. Proc. Natl. Acad. Sci. 111: 1583315838.

Unkefer, C.J., Sayre, R.T., Magnuson, J.K., Anderson, D.B., Baxter, I., et al. (2017) Review of the algal biology program within the National Alliance for Advanced Biofuels and Bioproducts. Algal Res. 22: 187-215.

Vonlanthen, S., Dauvillée, D., and Purton, S. (2015) Evaluation of novel starch-deficient mutants of Chlorella sorokiniana for hyper-accumulation of lipids. Algal Res. 12: 109-118.

Wang, C., Chen, X., Li, H., Wang, J., and Hu, Z. (2017) Artificial miRNA inhibition of phosphoenolpyruvate carboxylase increases fatty acid production in a green microalga Chlamydomonas reinhardtii. Biotechnol. Biofuels. 10: 91.

Wang, C., Li, Y., Lu, J., Deng, X., Li, H., and Hu, Z. (2018) Effect of overexpression of LPAAT and GPD1 on lipid synthesis and composition in green microalga Chlamydomonas reinhardtii. J. Appl. Phycol. 30: 1711-1719.

Wang, H.-W., Zhang, J.-S., Gai, J.-Y., and Chen, S.-Y. (2006) Cloning and comparative analysis of the gene encoding diacylglycerol acyltransferase from wild type and cultivated soybean. Theor. Appl. Genet. 112: 1086-1097.

Wang, H.-W., Zhang, B., Hao, Y.-J., Huang, J., Tian, A.-G., Liao, Y., et al. (2007) The soybean Dof-type transcription factor genes, GmDof4 and GmDof11, enhance lipid content in the seeds of transgenic Arabidopsis plants. Plant J. 52: 716-729.

Warakanont, J., Tsai, C.-H., Michel, E.J.S., Murphy, G.R., Hsueh, P.Y., Roston, R.L., et al. (2015) Chloroplast lipid transfer processes in Chlamydomonas reinhardtii involving a TRIGALACTOSYLDIACYLGLYCEROL 2 (TGD2) orthologue. Plant J. 84: 1005-1020.

Wichmann, J., Baier, T., Wentnagel, E., Lauersen, K.J., and Kruse, O. (2018) Tailored carbon partitioning for phototrophic production of $(\mathrm{E})$ - $\alpha$-bisabolene from the green microalga Chlamydomonas reinhardtii. Metab. Eng. 45: 211-222.

Work, V.H., Radakovits, R., Jinkerson, R.E., Meuser, J.E., Elliott, L.G., Vinyard, D.J., et al. (2010) Increased lipid accumulation in the Chlamydomonas reinhardtii sta 7-10 starchless isoamylase mutant and increased carbohydrate synthesis in complemented strains. Eukaryot. Cell. 9: 1251-1261.

Xie, B., Stessman, D., Hart, J.H., Dong, H., Wang, Y., Wright, D.A., et al. (2014) Highthroughput fluorescence-activated cell sorting for lipid hyperaccumulating Chlamydomonas reinhardtii mutants. Plant Biotechnol. J. 12: 872-882. 
Xue, J., Niu, Y.-F., Huang, T., Yang, W.-D., Liu, J.-S., and Li, H.-Y. (2015) Genetic improvement of the microalga Phaeodactylum tricornutum for boosting neutral lipid accumulation. Metab. Eng. 27: 1-9.

Xue, J., Wang, L., Zhang, L., Balamurugan, S., Li, D.-W., Zeng, H., et al. (2016) The pivotal role of malic enzyme in enhancing oil accumulation in green microalga Chlorella pyrenoidosa. Microb. Cell Factories. 15: 120.

Xue, J., Balamurugan, S., Li, D.-W., Liu, Y.-H., Zeng, H., Wang, L., et al. (2017) Glucose-6phosphate dehydrogenase as a target for highly efficient fatty acid biosynthesis in microalgae by enhancing NADPH supply. Metab. Eng. 41: 212-221.

Yamano, T., Iguchi, H., and Fukuzawa, H. (2013) Rapid transformation of Chlamydomonas reinhardtii without cell-wall removal. J. Biosci. Bioeng. 115: 691-694.

Yamaoka, Y., Achard, D., Jang, S., Legéret, B., Kamisuki, S., Ko, D., et al. (2016) Identification of a Chlamydomonas plastidial 2-lysophosphatidic acid acyltransferase and its use to engineer microalgae with increased oil content. Plant Biotechnol. J. 14: 21582167.

Yamasaki, T., Miyasaka, H., and Ohama, T. (2008) Unstable RNAi Effects Through Epigenetic Silencing of an Inverted Repeat Transgene in Chlamydomonas reinhardtii. Genetics. 180: 1927-1944.

Yang, W., Wittkopp, T.M., Li, X., Warakanont, J., Dubini, A., Catalanotti, C., et al. (2015) Critical role of Chlamydomonas reinhardtii ferredoxin-5 in maintaining membrane structure and dark metabolism. Proc. Natl. Acad. Sci. 112: 14978-14983.

Yoon, K., Han, D., Li, Y., Sommerfeld, M., and Hu, Q. (2012) Phospholipid:diacylglycerol acyltransferase is a multifunctional enzyme involved in membrane lipid turnover and degradation while synthesizing triacylglycerol in the unicellular green microalga Chlamydomonas reinhardtii. Plant Cell. 24: 3708-3724.

Zhang, R., Patena, W., Armbruster, U., Gang, S.S., Blum, S.R., and Jonikas, M.C. (2014) High-Throughput Genotyping of Green Algal Mutants Reveals Random Distribution of Mutagenic Insertion Sites and Endonucleolytic Cleavage of Transforming DNA. Plant Cell. 26: 1398-1409.

Zhu, Z., Yuan, G., Fan, X., Fan, Y., Yang, M., Yin, Y., et al. (2018) The synchronous TAG production with the growth by the expression of chloroplast transit peptide-fused ScPDAT in Chlamydomonas reinhardtii. Biotechnol. Biofuels. 11: 156.

Zorin, B., Hegemann, P., and Sizova, I. (2005) Nuclear-gene targeting by using singlestranded DNA avoids illegitimate DNA integration in Chlamydomonas reinhardtii. Eukaryot. Cell. 4: 1264-1272. 
Table 1. Summary of current uses of nuclease-directed genome editing in Chlamydomonas.

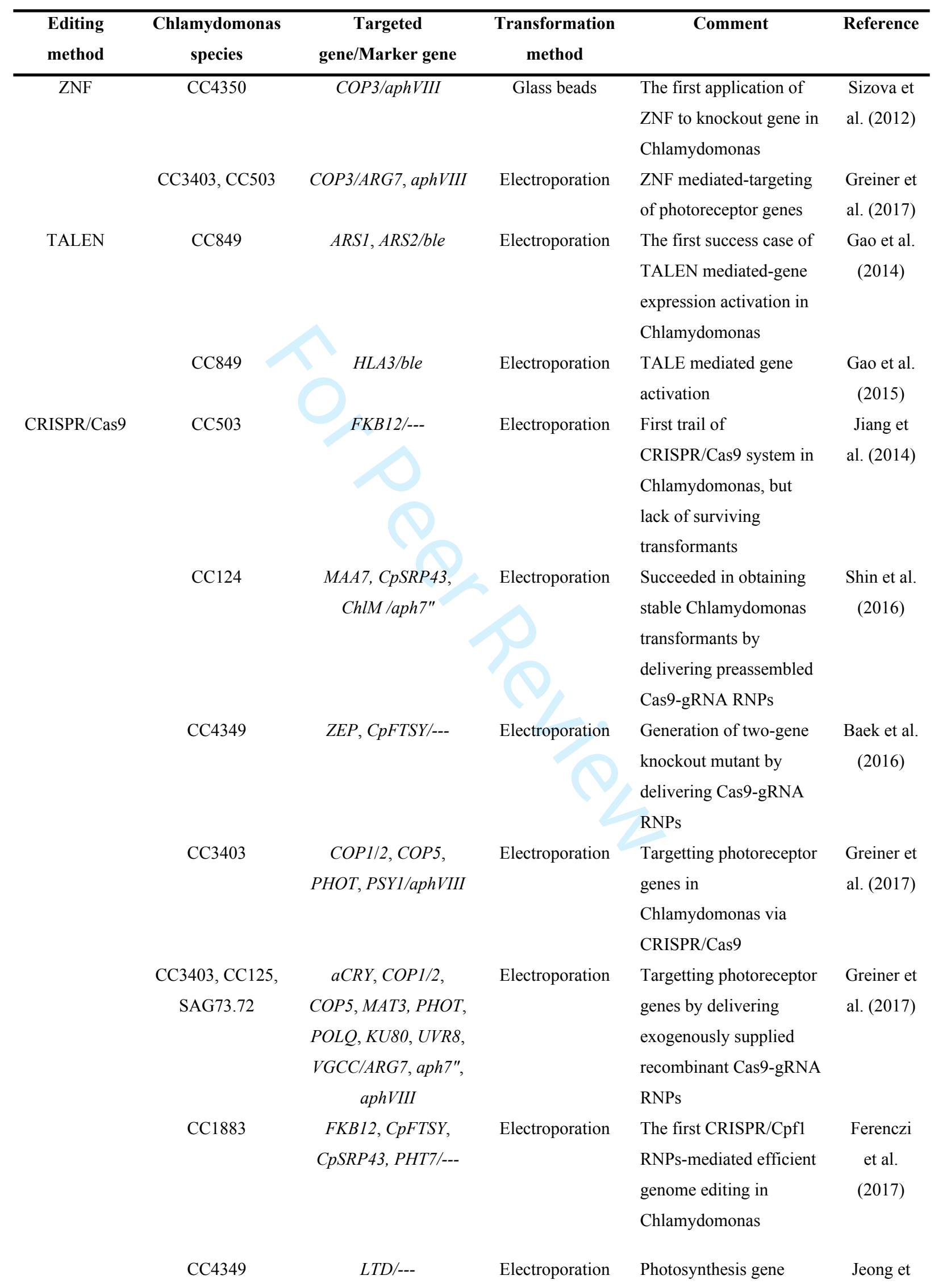




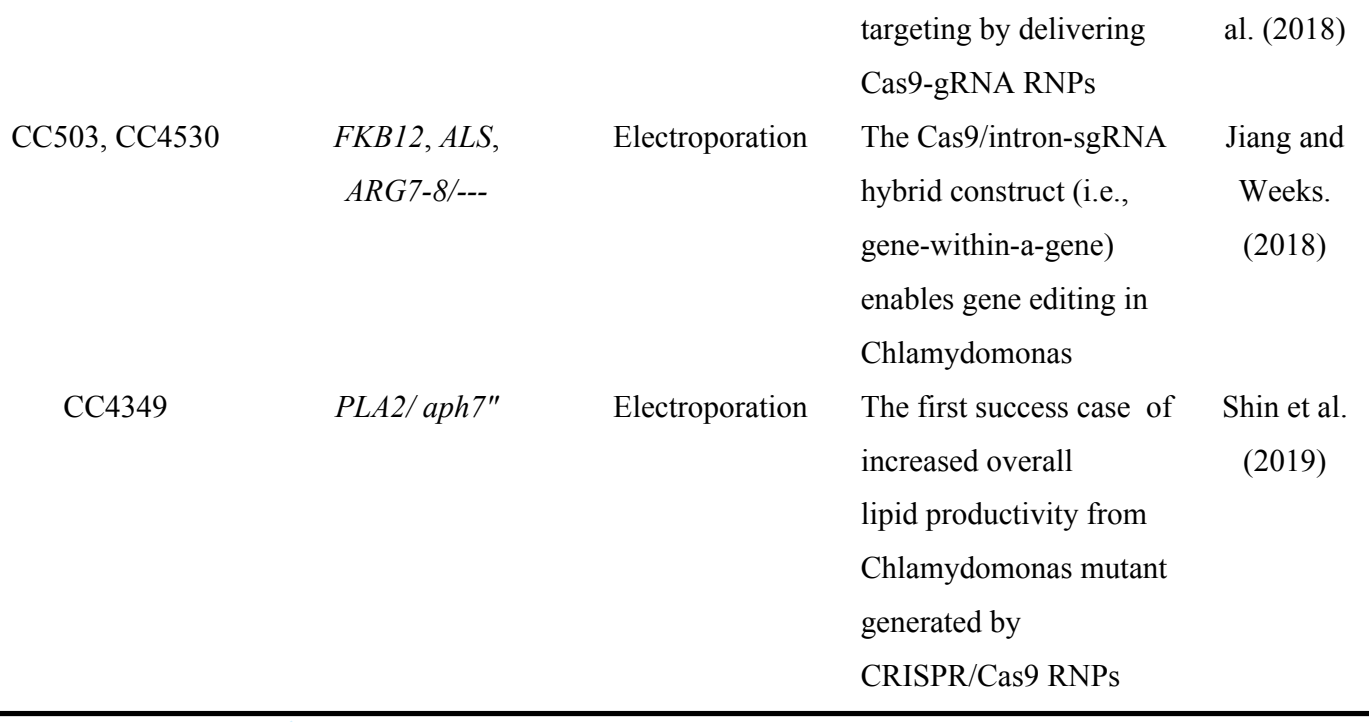

\section{Abbreviations:}

aCRY, animal-like cryptochrome; aph7", hygromycin B resistance gene; aphVIII, aminoglycoside 3'-phosphotransferase (paromomycin resistance gene); $A L S$, acetolactate synthase; $A R G$, argininosuccinate lyase; $A R S$, arylsulfatase; ble, bleomycin resistance gene; CRISPR/Cas9, clustered regularly interspersed palindromic repeats associated protein 9; COP, light-gated proton channel rhodopsin; $C p S R P$, chloroplast signal recognition particle; ChlM, Mg-protoporphyrin IX S-adenosyl methionine O-methyl transferase; CpFTSY, signal recognition particle receptor; $F K B 12$, peptidyl-prolyl cis-trans isomerase; HLA3 (also known as MRP1), a putative inorganic carbon transporter; KU80, ATP-dependent DNA helicase 2 subunit 2; LTD, light-harvesting chlorophyll- and carotenoid-binding proteins translocation defect gene; MAT3, retinoblastoma; MAA7, beta subunit of tryptophan synthase; PSY1, PHYTOENE SYNTHASE 1 ; PLA2, phospholipase A2; POLQ, DNA polymerase theta; PHOT, photoreceptor phototropin; PHT7, a putative phosphate transporter; TALEN, transcription activator-like effector nuclease; UVR8, a homomultimeric UV-B photoreceptor; $V G C C$, voltage-gated calcium channel; ZEP, zeaxanthin epoxidase, ZNF, zinc finger nuclease. “--." indicates selectable marker gene was not used. 


\section{Legends to figure}

Figure 1. The main approaches of genetic engineering of Chlamydomonas for altered oil content.

Abbreviations: amiRNAi, artificial microRNA interference; CRISPRi, clustered regularly interspaced short palindromic repeats interference; cht7, compromised hydrolysis of triacylglycerols 7; CrPEPC1/2, Chlamydomonas phosphoenolpyruvate carboxylase1/2; CrLPAAT1 and 2, Chlamydomonas lysophosphatidic acid acyltransferase 1 and 2; CrLIP1, Chlamydomonas lipase 1; cracx2, Chlamydomonas acyl-CoA oxidase 2; DOFs, Dof-type transcription factor genes; ER, endoplasmic reticulum; FA, fatty acid; $f d x 5$, ferredoxin5; icl, isocitrate lyase; lcs2, long-chain acyl-CoA synthetase; LD, lipid droplet; mldp, major lipid droplet protein; $m d h 2$, malate dehydrogenase 2 ; $n r r 1$, nitrogen-responsive regulator 1 ; OE, over-expressing strains; PSR1, phosphorus stress response 1; pgd1, plastid galactoglycerolipid degradation 1; pdat1, phospholipid:diacylglycerol acyltransferase 1; RNAi, RNA interference; std1, starch degradation 1; sta6 and 7; starch-less mutant 6 and 7; TAG, triacylglycerol; tgd2, trigalactosyldiacylglycerol 2; tarl, triacylglycerol accumulation regulator 1; vip1, a predicted diphosphoinositol pentakisphosphate kinase. 


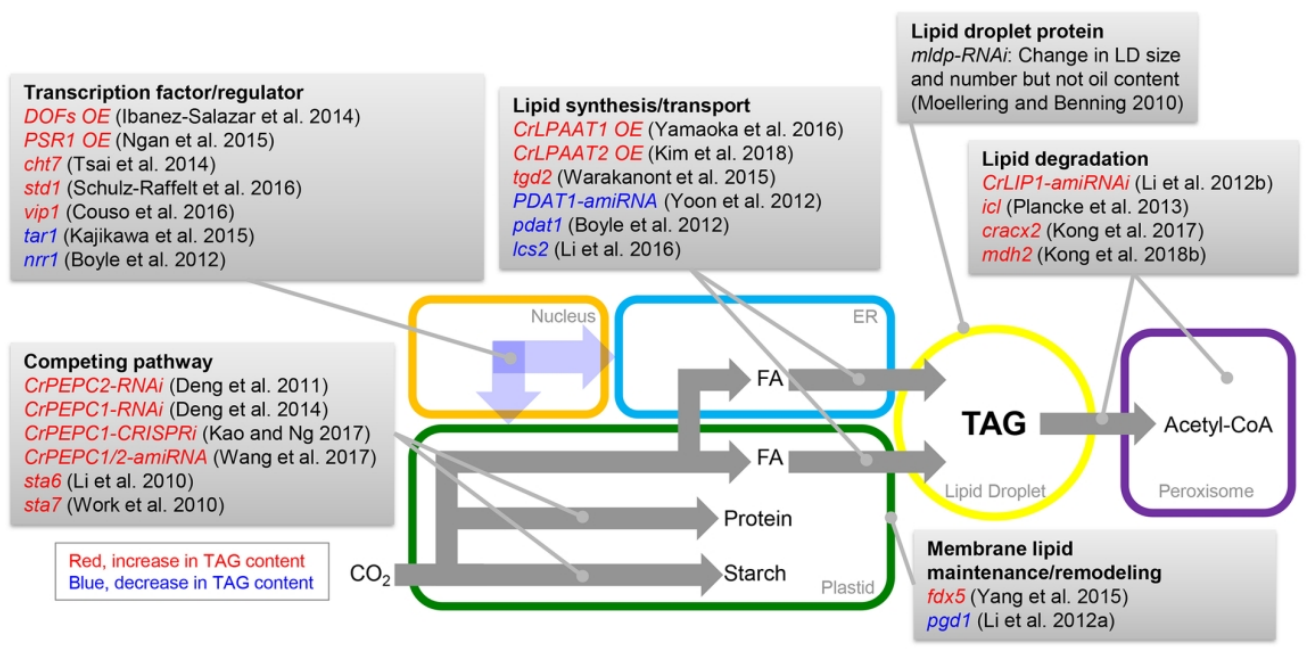

Figure 1. The main approaches of genetic engineering of Chlamydomonas for altered oil content.

$160 \times 79 \mathrm{~mm}(300 \times 300 \mathrm{DPI})$ 